\title{
Die Kritik der Politischen Ökonomie der Medien/Kommunikation: ein hochaktueller Ansatz
}

\author{
Christian Fuchs
}

Online publiziert: 13. Juni 2017

(C) Der/die Autor(en) 2017. Dieser Artikel ist eine Open-Access-Publikation.

Zusammenfassung Dieser Forums-Beitrag diskutiert die Aktualität des Ansatzes einer Kritik der Politischen Ökonomie der Medien/Kommunikation. Er analysiert den Status dieses Feldes, das international eine längere Tradition und Geschichte innerhalb der Medien- und Kommunikationswissenschaft hat. Seit dem Beginn der neuen Krise des Kapitalismus im Jahr 2008 hat das Interesse an Marx generell zugenommen. Zugleich ist die kommunikative und ideologische Dimension des unvorhersehbaren und turbulenten gesellschaftlichen Wandels in den Vordergrund getreten. Der Beitrag bietet eine kurze Einführung in einige Ansätze der Kritik der Politischen Ökonomie der Medien/Kommunikation. Mit der Diskussion von 14 Dimensionen wird hervorgehoben, dass der auf Marx zurückgehende komplexe, vielschichtige, offene, dynamische Theorie- und Forschungsansatz der Kapitalismusund Gesellschaftskritik in dieser gesellschaftlichen Situation für die Analyse des Zusammenhangs von Kommunikation und Gesellschaft hochaktuell ist. Somit ist die Kritik der Politischen Ökonomie ein fruchtbarer Ansatz zur empirischen und theoretischen Analyse und zur Aufklärung zeitgenössischer Kommunikation, der eine wahrhafte Praxisrelevanz hat.

Schlüsselwörter Kritik der Politischen Ökonomie $\cdot$ Kommunikation · Medien · Karl Marx · Kritische Theorie 


\section{Critique of the Political Economy of media/communication: a highly topical approach}

Abstract This debate article discusses how topical the approach of the Critique of the Political Economy of Media/Communication is today. The paper analyses the status of this field. At the international level, there is a longer tradition in the Critical Political Economy of Media/Communication, especially in the United Kingdom and North America. Since the start of the new crisis of capitalism in 2008, the interest in Marx's works has generally increased. At the same time communicative and ideological features of societal changes' unpredictable turbulences have become evident. This contribution introduces some specific approaches. It also discusses 14 aspects of why the complex, multidimensional, open and dynamic research approach of the critique of capitalism and society that goes back Marx's theory remains relevant today.

After an introduction (sect. 1), the article's second section provides a brief introduction to the critique of the political economy of media/communication by presenting the understandings of this field advanced by Peter Golding/Graham Murdock and Vincent Mosco. It also points out that there have been single representatives of the Critique of the Political Economy of Media/Communication in the Germanspeaking world, but that this approach is largely forgotten in German media and communication studies. The article provides a brief introduction to Horst Holzer's version of the critique of the political economy of media/communication: Holzer combined critical social theory and empirical social research in order to critically theorise and understand communication(s). He was critical of both systems theories of communication (e.g. Niklas Luhmann) and theories of communicative action (Jürgen Habermas) and worked out foundations of an alternative approach that are grounded in Marx's theory.

The third section argues that Karl Marx is not just a critic of capitalism, but that his approach can also help us to ground a critical theory of communication. It stresses that there are many elements in Marx's works that can help us to critically understand communication: critical journalism, limits on the freedom of the press, the analysis of the commodity form, the analysis of labour, exploitation, class, surplus-value, globalisation, crisis, modern technology, the General Intellect, communication, the means of communication, the contradiction between the productive forces and the relations of production, dialectics, ideologies, social struggles, and democratic alternatives.

Sect. 4 provides an example of how to use the approach of the Critique of Political Economy for analysing concrete communication phenomena. After the 2011 Arab Spring, there were many discussions about the role of digital and social media in protests. Some observers claimed that we had experienced Facebook and Twitter revolutions. Others argued that such claims are technologically deterministic and that protests would not be a matter of communications, but of crowds gathering in the streets and occupying squares. Using the critique of the Political Economy of Media/Communication as framework, the OccupyMedia!-study analysed how activists used social media and how capitalist power and state power limited protest communication. It also explored the potential of alternative digital media in protest 
and the challenges that political economy posed for the establishment and use of such communications.

The article concludes that the Critique of the Political Economy of Media/ Communication is a fruitful, praxis-oriented approach for the empirical and theoretical analysis of contemporary communication(s). In the German-speaking world and in German media and communication studies, there has been unjustified fear of Marx. In addition, examples from the 1970s until today show that representatives of the Critique of the Political Economy of Media/Communication in the Germanspeaking world have had justified fears over being considered as Marxists.

The future will show if new developments and attempts to advance the Critique of the Political Economy of Media/Communication in the German-speaking world will make a difference that makes a difference or not.

Keywords Critique of Political Economy - Communication · Media - Karl Marx · Critical Theory

\section{Einleitung}

Unter der Kritik der Politischen Ökonomie der Medien/Kommunikation ist ein Ansatz zu verstehen, der Theorie, Philosophie und empirische Forschung anwendet, um den Zusammenhang von Kommunikation, Kapitalismus, Herrschaft und Macht $\mathrm{zu}$ analysieren, kritisch zu reflektieren und dadurch Wissen zu generieren, das eine bedeutende Rolle in der gesellschaftlichen Praxis, die auf die Veränderung der Welt abzielt, spielen kann. „Zu den Grundfragen einer kommunikationswissenschaftlichen Medienökonomie als Kritik der Politischen Ökonomie der Medien gehört die Analyse des Verhältnisses von Medien und kapitalistischer Gesellschaft, also die Rolle der Medien für das gesamte materielle, wirtschaftliche, gesellschaftliche, soziale, politische und kulturelle menschliche Leben." (Knoche 2002, S. 105)

Die Kritik der Politischen Ökonomie ist einer der Ansätze innerhalb der politischen Ökonomie der Medien/Kommunikation. Andere Formen gehen auf die neoklassische Wirtschaftslehre, den Neo-Keynesianismus, den Institutionalismus, die feministische politische Ökonomie, die politische Ökologie und weitere Ansätze zurück (vgl. Mosco 2009; Hardy 2014; Winseck 2011). Diese Ansätze überlappen teilweise einander und sind nicht klar voneinander abgrenzbar. Dennoch kann man allgemein zwischen orthodoxen Ansätzen, die neoklassisch, neoliberal und neokonservativ sind, und heterodoxen Ansätzen unterscheiden.

Dieser Diskussionsbeitrag beschäftigt sich mit der Relevanz der Kritik der Politischen Ökonomie der Medien/Kommunikation in der heutigen Gesellschaft. Abschn. 2 bietet eine kurze Einführung in das Feld der Kritik der Politische Ökonomie der Medien/Kommunikation. Abschn. 3 setzt sich mit der Bedeutung der Marx'schen Theorie für die Medien- und Kommunikationswissenschaft auseinander. Abschn. 4 präsentiert ein Anwendungsbeispiel der Kritik der Politischen Ökonomie der Medien/Kommunikation, nämlich die Analyse der politischen Ökonomie der Alternativmedien und sozialer Bewegungen. 


\section{Das Forschungsfeld der Kritik der Politischen Ökonomie der Medien/ Kommunikation}

Die auf Marx zurückgehenden Ansätze der Kapitalismuskritik, die mit den Begriffen „Kritik der Politischen Ökonomie“ und „Kritische Politische Ökonomie“ zusammengefasst werden, haben eine lange Tradition und eine relativ große Anzahl von Ausprägungen (vgl. Bidet und Kouvelakis 2008). Diese Ansätze unterscheiden sich hinsichtlich ihres Gegenstandsbereiches, der Art der Theoriebildung und -ausrichtung, der Rolle empirischer und theoretischer Ansätze, des Verhältnisses von Struktur und Handlung und des Verhältnisses von Ökonomie und Gesellschaft. Sie haben aber auch die Gemeinsamkeit, dass sie den analysierten Gegenstandsbereich immer im Zusammenhang mit kapitalistischer Herrschaft, Machtstrukturen, Klassen, Klassenkämpfen, Formen der Produktion und Reproduktion und alternativen Gesellschaftsformationen sehen. Von Marx zu sprechen bedeutet daher nicht die Fixierung auf eine Person oder ein Buch, sondern die Orientierung an einer komplexen, vielschichtigen, offenen und sich dynamisch entwickelnden Theorie- und Forschungstradition. Will man Marx neu entdecken, so gibt es dazu viele Ansatzpunkte, Möglichkeiten und Theorieversionen.

Die Ausprägung des Interesses an der von Marx beeinflussten Kapitalismuskritik und kritischen Gesellschaftsanalyse steht in komplexer Weise in Zusammenhang mit der gesellschaftlichen Entwicklung und den Resultaten der gesellschaftlichen Kämpfe und Klassenkämpfe. Während und unmittelbar nach der 1968er-Studentenbewegung war dieses Interesse relativ stark ausgeprägt. Durch den Aufstieg des neoliberalen Kapitalismus, den Zusammenbruch des „,real existierenden Sozialismus“ und postmoderne Ansätze, die die von Marx beeinflusste kritische Gesellschaftsanalyse als totalitäre ,große Erzählung“ verwarfen, sich der Mikroanalyse und Mikropolitik widmeten und an ein Ende der Geschichte glaubten, ging dieses Interesse deutlich zurück. Die neue Weltwirtschaftskrise, die im Jahr 2008 begann, die Krisen nationaler und transnationaler Staatsmacht (inklusive der Europäischen Union) in Folge von Austeritätsmaßnahmen und kurzsichtigen Reaktionen auf Flüchtlinge und Krieg, die politische und ideologisch Krise des neoliberalen Regulationsmodells, die soziale Krise, die sich durch prekäre Lebens- und Arbeitsverhältnisse auszeichnet und von der in vielen Teilen der Welt vor allem junge Menschen betroffen sind - sie alle haben zu einer Legitimationskrise des Kapitalismus geführt. Im Zuge dieser Krise hat das Interesse an der von Marx beeinflussten Kapitalismus- und Gesellschaftskritik deutlich zugenommen.

Tab. 1 Durchschnittliche jährliche Anzahl der Nennung kapitalismuskritischer Kategorien in den Titeln von gesellschaftswissenschaftlichen Zeitschriftenartikeln in bestimmten Zeitperioden. Datenquelle: Social Sciences Citation Index (aufgerufen am 24. März 2017; Schlagwortsuche: Marx* OR capitalis* OR commodi*)

\begin{tabular}{ll}
\hline Zeitraum & $\begin{array}{l}\text { Anzahl der durchschnittlichen Erwähnungen pro } \\
\text { Jahr }\end{array}$ \\
\hline $1970-1979$ & 535 \\
$1980-1989$ & 822 \\
$1990-1999$ & 506 \\
$2000-2009$ & 433 \\
$2010-2016$ & 713 \\
\hline
\end{tabular}


Diese theoretische Analyse lässt sich für die Gesellschaftswissenschaften empirisch fundieren. Tab. 1 zeigt für verschiedene Zeitperioden die Anzahl der Erwähnung von kapitalismuskritischen Stichwörtern in den Titeln gesellschaftswissenschaftlicher Zeitschriftenartikel, die im Social Sciences Citation Index indiziert sind. In den 1970er- und 1980er-Jahren war die Anzahl deutlich höher als in den 1990ern und der ersten Dekade des neuen Millenniums. Seit 2010 hat die jährliche Durchschnittszahl wieder deutlich zugenommen, nämlich nahezu auf das Niveau der 1980er-Jahre. Die turbulenten gesellschaftlichen Zeiten, in denen wir leben, haben in den Gesellschaftswissenschaften offenbar zu einem Anstieg des Interesses an und der Beschäftigung mit der Kritik der Politischen Ökonomie geführt.

Die Kritik der Politischen Ökonomie der Medien/Kommunikation hat sich vor allem international und in Großbritannien, den USA, Kanada und Lateinamerika zu einem gewissen Grad institutionalisiert (vgl. Wasko 2014; Mosco 2009). In der International Association for Media and Communication Research (IAMCR) existiert seit 1978 eine sehr aktive Political Economy-Sektion. Es gibt wissenschaftliche Zeitschriften wie tripleC: Communication, Capitalism \& Critique oder The Political Economy of Communication, ferner Konferenzen, Lehrbücher und -veranstaltungen, Sammelbände, Handbücher, Forschungs- und Dissertationsprojekte etc.

Vincent Mosco (2009, S. 2) definiert den Ansatz der politischen Ökonomie der Medien/Kommunikation als die ,Analyse der wechselseitigen Konstituierung von gesellschaftlichen Beziehungen, insbesondere der Machtverhältnisse, und der Produktion, Distribution und Konsumtion von Ressourcen, inklusive kommunikativer Ressourcen“. Laut Mosco geht es bei einer Analyse von Kommunikationsphänomenen mit Hilfe des Ansatzes der Kritik der Politischen Ökonomie darum, den Untersuchungsgegenstand in Beziehung zu setzen zu Prozessen der Kommodifizierung (von Inhalten, Arbeitskraft und Publikum), der Globalisierung und Internationalisierung, Privatisierung, Liberalisierung, Kommerzialisierung, zu Konzentrationsprozessen (horizontaler und vertikaler Integration, strategischen Allianzen, Joint Ventures), zu Strukturierungsprozessen (z. B. zu Klassenverhältnissen, Rassismus, Geschlechterbeziehungen) und gesellschaftlicher Praxis (sozialen Bewegungen, gesellschaftlichem Wandel oder Protestbewegungen).

Graham Murdock und Peter Golding betonen, dass kapitalistische Medien Information als Ware verkaufen und/oder als Werbeplattformen in die Gesamtwirtschaft eingebunden sind (vgl. Murdock und Golding 1974). Das Spezifikum der Medien bestehe darin, dass sie Ideen über Wirtschaft und Politik öffentlich kommunizieren, wodurch neben der Analyse der Rolle der Medien im Kapitalismus auch die Ideologiekritik eine zentrale Aufgabe der Kritik der Politischen Ökonomie der Medien/Kommunikation darstelle. Die Kritik der Politischen Ökonomie der Medien/ Kommunikation unterscheide sich in vierfacher Hinsicht von Mainstreamansätzen: „Erstens ist dieser Ansatz holistisch. Zweitens ist er historisch. Drittens ist die Auseinandersetzung mit dem Verhältnis von kapitalistischen Unternehmen und öffentlicher Regulation zentral. Und letztlich, und vielleicht am wichtigsten, geht der Ansatz über technische Fragen der Effizienz hinaus und setzt sich mit grundlegenden moralischen Fragen der Gerechtigkeit, der Fairness und des öffentlichen Wohls auseinander." (Murdock und Golding 2005, S. 61) Man kann also sagen, dass die Kritik der Politischen Ökonomie der Medien/Kommunikation dadurch materialistisch ist, 
dass sie Technik- und Medienzentriertheit vermeidet und „die Medien der Kommunikation dezentriert“ (Mosco 2009, S. 66), diese also immer im Zusammenhang mit gesamtgesellschaftlichen Verhältnissen betrachtet.

In der Auseinandersetzung zwischen Kritischer Politischer Ökonomie und Cultural Studies ging es um die Frage, welche Rolle Struktur/Handlung, Makro/Mikro, Gesellschaftswissenschaft/Geisteswissenschaft, Herrschaft/Widerstand, Produktion/ Konsum, Wirtschaft/Kultur, Ausbeutung/Macht und Klasse/Identität bei der Analyse von Kommunikationsphänomen spielen und wie das Verhältnis dieser Kategorien zu fassen ist. Heute hat sich international stärker der Ansatz durchgesetzt, dass beide Seiten dieser Widersprüche dialektisch und integrativ zu behandeln sind. Dieser Umstand wird zum Beispiel im letzten Interview, das Stuart Hall vor seinem Tod gegeben hat, deutlich, in dem er sagte, dass man ,zur politischen Rolle der Cultural Studies zurückkehren sollte, der politischen Dimension der Cultural Studies, und man sollte sich dabei fragen: ,Wenn die Wirtschaft nicht alles in letzter Instanz determiniert, was ist dann die Rolle der Wirtschaft in der Reproduktion des materiellen und symbolischen Lebens?` Man muss sich ökonomische Fragen stellen. [...] Es gibt eine Art Rückkehr. [...] Gramsci sagte immer, dass die Wirtschaft niemals vergessen werden sollte. [...] Es wird euch nicht überraschen zu erfahren, dass ich denke, diese Rückkehr sollte mehr eine Rückkehr dazu sein, was Cultural Studies hätten sein sollen und in der Anfangsphase waren. Die Cultural Studies haben sich irgendwie verirrt.“ (Jhally 2016, S. 337-338) Hall plädiert dafür, dass sich die Cultural Studies stärker mit der „,marxistischen Tradition des kritischen Denkens“ (Jhally 2016, S. 338) auseinandersetzen.

Horst Holzer (1994, S. 185) sprach von dem Umstand, dass es sich bei der Kritik der Politischen Ökonomie der Medien/Kommunikation im deutschen Sprachraum um eine ,vergessene Theorie“ handle. Es hat durchwegs Vertreter mit ausgezeichneten Ansätzen gegeben, wie zum Beispiel Holzer, wie Manfred Knoche, Dieter Prokop, Jörg Becker, Wulf Hund, Bärbel Kirchhoff-Hund, Franz Dröge, Jörg Aufermann, Rudi Schmiede, Lothar Bisky, Jürgen Alberts, u. a. Mit Ausnahmen, wie der Professur von Manfred Knoche in Salzburg (1994-2009), hat sich der Ansatz der Kritik der Politischen Ökonomie aber in der deutschsprachigen Medien- und Kommunikationswissenschaft nicht institutionalisiert.

Horst Holzer kombinierte kritische Gesellschaftstheorie und empirische Sozialforschung mit der Analyse von Medien und Kommunikation. Er vertrat also den Ansatz einer Kritik der Politischen Ökonomie der Medien/Kommunikation, der auf einer Dialektik von Theorie und Empirie beruht. So verwendete Holzer zum Beispiel die Inhaltsanalyse, die Sekundäranalyse von empirischen Studien und die Analyse von makroökonomischen Daten, um den Zusammenhang von Kommunikation, Ökonomie und Demokratie zu analysieren (vgl. Holzer 1971). Er betonte ähnlich wie Habermas, dass Werbung, Medienkonzentration, die kommerzielle Orientierung der Medien, ,die Personalisierung gesellschaftlicher Tatbestände“ und die „,betonte Vermischung von individuellen Lebensproblemen und öffentlichen Angelegenheiten“ (Holzer 1971, S. 151) den demokratischen Charakter der Öffentlichkeit unterminierten. Der Unterschied zwischen Habermas und Holzer besteht darin, dass Letzterer nicht rein gesellschaftstheoretisch argumentiert, sondern empirische Resultate ge- 
sellschaftskritisch und auf Basis einer kritischen Theorie der Kommunikation und der Gesellschaft interpretiert.

Holzers dialektische Gesellschafts- und Kommunikationstheorie entstand als Kritik an der Handlungs- und der Systemtheorie. An der Systemtheorie, die entscheidend von Talcott Parsons geprägt wurde, kritisierte er, dass diese soziale Systeme als Subjekt fasse (vgl. Holzer 1971, S. 255) und „die Hypostasierung eines realgesellschaftlichen Status quo zur Ordnung von Gesellschaft schlechthin“ (Holzer 1971, S. 250) betreibe. Luhmanns Systemtheorie der Kommunikation sei nicht imstande, den Zusammenhang von Kommunikation, Körper und Psyche zu erhellen und ,die Verschränkung von Genese, Beschaffenheit und Funktion der Massenkommunikation“ (Holzer 1994, S. 182) mit Medienproduktion, Medienorganisationen, Medieninhalten, Mediennutzung und -rezeption und menschlichem Bewusstsein zu erklären.

An Habermas' Theorie des kommunikativen Handelns kritisierte Holzer, dass diese das Verhältnis von Arbeit und Interaktion nicht dialektisch, sondern dualistisch fasste (vgl. Holzer 1987). Habermas ist „,nicht imstande, die wesentliche Bestimmung der gesellschaftlichen Produktion zu erkennen: daß im Prozeß der Produktion eben nicht nur die Produktivkräfte entwickelt werden, sondern auch die gesellschaftlichen Beziehungen - eingeschlossen: ,Kommunikation', ,Interaktion “-, die die Menschen in diesem Produktionsprozeß miteinander eingehen" (Holzer 1987, S. 27). Georg Lukács hat im Unterschied zu Habermas in seiner Ontologie des gesellschaftlichen Seins den gesellschaftlichen Produktionsprozess, der auf einer Dialektik von Arbeit und Kommunikation beruht, als teleologische Setzung charakterisiert (vgl. Fuchs 2016a, Kap. 2).

Holzer arbeitete an einer auf der Marx'schen Gesellschaftstheorie beruhenden materialistischen Theorie der Kommunikation. Es bestehe eine Dialektik von Arbeit und Kommunikation. Erkenntnis und Kommunikation seien „Zwei Seiten des Prozesses“, der „,den gesellschaftlich organisierten Stoffwechsel mit der Natur und die innergesellschaftlichen Auseinandersetzungen regelt“ (Holzer 1973, S. 57). Entscheidend für eine derartige Theorie ist nun, dass sie Kritik der Politischen Ökonomie der Medien/Kommunikation ist. Holzer analysierte Kommunikation und Medien im Kapitalismus auf der Ebene der Einzelkapitalien und des Gesamtkapitals (vgl. Holzer 1973, S. 129-137, 1994, S. 202-204). Auf der Ebene der Einzelkapitalien hat das Medien- und Kommunikationssystem eine Kapitalökonomie, in der Information direkt eine Form der Kapitalverwertung und Mehrwertproduktion ist, und eine warenzirkulierende Rolle bei der „Verbreitung von ,Konsumklima“ und ,Bewerbung" spezifischer Produkte und Dienstleistungen" (Holzer 1994, S. 202). Auf der Ebene des Gesamtkapitalismus hat das Kommunikationssystem eine ideologische Rolle ,zur Sicherung und Legitimation der Kapitalherrschaft“" (Holzer 1973, S. 131) und „des gesellschaftlichen Ordnungsprinzips“ (Holzer 1994, S. 202) sowie eine reproduktive Rolle als Informations- und Unterhaltungsquelle, die zur „Herstellung, Erhaltung und Wiederherstellung" (Holzer 1973, S. 131) der Arbeitskraft diene.

Die Kritik der Politischen Ökonomie der Medien/Kommunikation ist im deutschsprachigen Raum bisher institutionell gescheitert und wurde institutionell verhindert. Die Auffassung, dass es sich bei der kritischen politischen Ökonomie um einen wichtigen theoretischen und empirischen Beitrag und Ansatz zur Erforschung von 
Medien und Kommunikation handelt, hat sich nicht durchgesetzt. Obwohl die Kritik der Politischen Ökonomie der Medien/Kommunikation heute höchst relevant ist, ist sie im deutschsprachigen Raum vergessen und wenig etabliert. Einer der Einflussfaktoren wird am Beispiel Horst Holzers deutlich, gegen den 1971 wegen seiner DKP-Mitgliedschaft bei der Berufung auf eine Professur in Bremen ein Berufsverbot verhängt wurde, was auch zur Verhinderung seiner Verbeamtung an der LMU München im Jahr 1974 und zum Ende seiner akademischen Karriere führte (vgl. Bönkost 2011; Scheu 2012).

Die politische Jagd auf Marxistinnen und Marxisten ist mehr als 45 Jahre nach Holzers Berufsverbot immer noch nicht zu Ende. Medien- und Kommunikationswissenschaftler sind auch heute noch davon betroffen: Im Herbst 2012 leitete der Bayerische Verfassungsschutz eine Prüfung ein, als Kerem Schamberger eine finanzierte Doktorandenstelle im Bereich der Medien- und Kommunikationswissenschaft an der LMU München angeboten wurde. Schamberger ist in Bayern als linker Aktivist bekannt. Er schildert die Umstände folgendermaßen:

Als ich mich im Juli 2016 für die Stelle als Wissenschaftlicher Mitarbeiter am Lehrbereich Meyen beworben habe und genommen wurde, musste ich einen sogenannten ,Bogen zur Prüfung der Verfassungstreue" ausfüllen. Dort sind Organisationen aufgelistet, die nach Meinung des bayerischen Verfassungsschutzes verfassungsfeindlich sind. Dieser ,Gesinnungs'-Bogen muss von allen Bewerbern des öffentlichen Dienstes in Bayern ausgefüllt werden. Skandalöserweise finden sich dort antifaschistische Organisationen, wie die Vereinigung der Verfolgten des Naziregimes (VVN), direkt neben Naziorganisationen wie der NPD oder militanten faschistischen Kameradschaften. Auch kapitalismuskritische, linke Organisationen, wie die DKP oder die Rote Hilfe sind dort aufgelistet. Wenn man dort nun ankreuzt irgendwo Mitglied zu sein, dann wird der Verfassungsschutz um Auskunft gebeten, was es mit der Person auf sich hat. So war das auch in meinem Fall. [...] Angekreuzt habe ich die Deutsche Kommunistische Partei (DKP), die Vereinigung der Verfolgten des Naziregimes - Bund der AntifaschistInnen (VVN), die Rote Hilfe und die Sozialistische Deutsche Arbeiterjugend (SDAJ), in der ich bis 2013 Mitglied war. (Schamberger 2017, S. 93, 94)

Die LMU München stellte Schamberger schließlich trotz eines negativen Gutachtens ein, da die Universität keine Zweifel an seiner Verfassungstreue und seinem Eintreten für die demokratische Form des Sozialismus hatte.

\section{Karl Marx als Kapitalismuskritiker und Kommunikationstheoretiker}

Marx war ein historischer und dialektischer Denker. Da sich die Gesellschaft wandelt, müssen sich auch die Kategorien wandeln, mit denen diese analysiert wird. Daher sollten gesellschaftstheoretisch jedoch zwei Extreme vermieden werden. Nämlich einerseits die Annahme, dass wir heute in einer radikal neuen, postmodernen digitalen oder Informationsgesellschaft leben. Und andererseits die Prämisse, dass sich die Gesellschaft seit dem 19. Jahrhundert gar nicht verändert habe. Beide Her- 
angehensweisen können durch den dialektischen Ansatz der Gesellschaftsanalyse vermieden werden, der von einer Dialektik von Kontinuität und Diskontinuität in der Entwicklung ausgeht. Eine neue Phase der gesellschaftlichen und kapitalistischen Entwicklung hebt ältere Phasen auf, d.h. zu einem bestimmten Grad wird das Bestehende bewahrt, eliminiert oder durch neue Qualitäten ergänzt. Dieser Grad ist bestimmt durch eine Dialektik von Zufall und Notwendigkeit, Strukturen und Handeln, Krisen und gesellschaftlichen Kämpfen.

Wir leben heute in einem Kapitalismus, der wie im 19. Jahrhundert auf der Ausbeutung der Arbeit beruht. Kapitalistischer Wandel hat aber zugleich zu Veränderungen innerhalb des Kapitalismus geführt, der heute als eine Vielzahl ineinandergreifender Kapitalismen organisiert ist, zum Beispiel als Finanzmarktkapitalismus, digitaler Kapitalismus, Wissens- und Inforationskapitalismus, neoliberaler Kapitalismus, autoritärer Kapitalismus, Mobilitätskapitalismus, hyperindustrieller Kapitalismus, usw.

Die Beschäftigung mit der Kritik der Politischen Ökonomie ist heute in vielerlei Hinsicht für die kritische Analyse der Medien und der Kommunikation von Bedeutung (vgl. Fuchs 2017a, 2017b, 2016a, 2016b, 2015, 2014a, 2011, 2008; Fuchs und Mosco 2017a, 2017b, 2012). Sie geht auf Marx' Analyse des Kapitalismus und der Gesellschaft zurück.

Ein erster interessanter Aspekt an der Kritik der Politischen Ökonomie ist der Umstand, dass Marx ein Journalist und Intellektueller war, der sich mit den politischen Entwicklungen seiner Zeit umfassend kritisch auseinandersetzte. In Zeiten der Erosion des investigativen Journalismus erinnert uns die Marx'sche journalistische Praxis an eine Zeit, in der die Kommerzialisierung und Kapitalisierung der Medien und damit die Kolonialisierung der Öffentlichkeit weniger weit vorangeschritten war. Journalismus war für Marx ein Mittel der Gesellschaftskritik.

Marx war ein glühender Verfechter der Demokratie, der Presse- und der Meinungsfreiheit. Die Gefahr der Beschränkung dieser Freiheit sah er einerseits durch staatliche Zensur sowie durch Kapitalisierung und Medienkonzentration gegeben: „Die erste Freiheit der Presse besteht darin, kein Gewerbe zu sein.“ (Marx 1842, S. 71) Heute leben wir in einer Welt, in der es starke Kapitalkonzentration nicht nur im traditionellen Mediensektor gibt, sondern auch bei den neuen digitalen Medien. Google hat das Kapital im Bereich der Suchmaschinen monopolisiert, Facebook im Bereich der sozialen Netzwerke, Amazon im Onlinehandel, Microsoft im Betriebssystem, etc. Die Mechanismen, mit denen Kapitalkonzentration und Kommodifizierung operieren, und die Formen, in denen sie sich ausdrücken, haben sich mit dem Wandel zum digitalen Kapitalismus verändert, die Phänomene der Medienkonzentration und der Medienmonopole bleiben jedoch grundlegende Strukturprinzipien des Kapitalismus. Google hat im Markt der Onlinewerbung eine dominante Stellung. Dies ist ein globaler Markt, da das Internet ein globales Informations- und Kommunikationsmittel ist. Laut Schätzungen kontrollierte Google im Jahr 2016 $71 \%$ der Onlinesuche. ${ }^{1}$ Google-Suche und Google-Werbung sind algorithmisch. Sie beruhen auf einer konstanten Überwachung, Speicherung und Auswertung (fast) aller Onlineaktivitäten aller Nutzer, wodurch Big Data generiert und kommodifiziert

\footnotetext{
1 http://www.netmarketshare.com, zugegriffen am 31. Dezember 2016.
} 
wird. Die Online-Kapitalkonzentration operiert also in einem globalen Markt, der auf Algorithmen und Big Data basiert.

Die Analyse der Warenform als Elementarform des Kapitalismus ist ein zweiter interessanter Aspekt der Kapitalismuskritik. Marx beginnt das erste Kapitel im ersten Band von Das Kapital mit den Worten: „Der Reichtum der Gesellschaften, in welchen kapitalistische Produktionsweise herrscht, erscheint als eine , ungeheure Warensammlung ', die einzelne Ware als seine Elementarform.“ (Marx 1867, S. 49)

Die politisch-ökonomische Strategie des Kapitalismus besteht darin, immer weitere Bereiche der Gesellschaft unter die Warenform zu subsumieren. Dabei wurde in den letzten Jahrzehnten vor allem nicht Halt gemacht vor Bereichen, die traditionell von der Kommodifizierung geschützt oder verschont waren, wie öffentlich-rechtliche Dienste (inklusive der öffentlich-rechtlichen Medien, Bildung, Erziehung, Gesundheit und Universitäten), dem menschlichen Körper, dem menschlichen Geist, der Kommunikation, der Natur usw. Im Bereich der digitalen Medien hat die Kommodifizierung die Arbeitskraft (digitale Arbeit), digitale Inhalte, digitale Technologien, den Zugang zu Plattformen, das Online-Publikum, die Online-Prosumenten (produzierende Konsumenten und Konsumentinnen) und Big Data erfasst.

Die Kategorien der Ausbeutung der Arbeit, des Mehrwerts und der Klassenverhältnisse sind ein dritter aktueller Aspekt der Kritischen Politischen Ökonomie. Im Kapitalismus gilt der Arbeiter ,nur als Maschine zur Produktion von Mehrwert“ und „der Kapitalist nur als Maschine zur Verwandlung dieses Mehrwerts in Mehrkapital““ (Marx 1867, S. 621). Durch die historische Differenzierung des Kapitalismus ist die Klassenstruktur komplexer geworden (vgl. Wright 1997; Negri 2017, 1982/1988; Federici 2012; Dalla Costa und James 1973; Smythe 1977). Dies zeigt sich an der Entstehung von Managern, unbezahlten Praktikanten, an Prekariat, Freiberuflern, Wissensarbeiterinnen, digitaler Arbeit usw. Die Produktionsverhältnisse des digitalen Kapitalismus beruhen auf einer internationalen Arbeitsteilung, in der verschiedene Ausbeutungsformen (wie Sklavenarbeit, digitale Hausarbeit, tayloristische Industriearbeit, prekäre digitale Arbeit, die Arbeit hochbezahlter und überarbeiteter Softwareingenieure, unbezahlte digitale Schattenarbeit, niedrig bezahlte digitale Arbeit in Entwicklungsländern) zusammenwirken.

Die Globalisierung des Kapitals stellt einen vierten aktuellen Aspekt der Kritik der Politischen Ökonomie dar. Marx betonte, dass der Kapitalismus die grundlegende Tendenz hat, sich räumlich auszudehnen, um Märkte, Produktions- und Konsumtionssphären für Waren, Arbeitskräfte und Kapital zu schaffen. „Das Bedürfnis nach einem stets ausgedehnteren Absatz für ihre Produkte jagt die Bourgeoisie über die ganze Erdkugel. Überall muss sie sich einnisten, überall anbauen, überall Verbindungen herstellen.“ (Marx und Engels 1848, S. 465). In den letzten Jahrzehnten hat der Kapitalismus die Produktion von Waren und Mehrwert transnationalisiert und den Kapitalexport ähnlich wie zu Beginn des 20. Jahrhundert stark vorangetrieben. Eine Konsequenz davon war, dass man seit den 1990er-Jahren in öffentlichen Diskussionen und den Gesellschaftswissenschaften sehr viel von Globalisierung gesprochen hat.

Diese Begrifflichkeit ist nicht falsch, aber die auf Marx beruhende Gesellschaftskritik hat den Vorteil, dass sie die spezifische Form der kapitalistischen Globalisierung als Imperialismus gefasst hat. In neueren Diskussionen hat zum Beispiel David 
Harvey den Begriff des neuen Imperialismus geprägt (vgl. Harvey 2005), um die Kombination von Finanzialisierung und Neoliberalismus als Charakteristikum der heutigen kapitalistischen Globalisierung hervorzuheben. Der ökonomische Sektor des Medien-, Informations-, Kommunikations-, Kultur- und digitalen Kapitals ist einer derartigen kapitalistischen Globalisierungstendenz unterworfen. Im Jahr 2016 befanden sich unter den 30 weltgrößten transnationalen Konzernen sieben derartige Unternehmen: Apple (\#8), AT\&T (\#12), Verizon Communication (\#15), China Mobile (\#18), Samsung Electronics (\#18), Microsoft (\#23), Google/Alphabet (\#27). ${ }^{2}$ Unter diesen 30 Unternehmen befinden sich 15 Finanzunternehmen (Banken, Versicherungen) und sieben Energie- und Mobilitätsfirmen (Ö1, Gas, Energieversorgung, Autoproduktion). Dieser Umstand weist darauf hin, dass der Kapitalismus heute eine Kombination von Finanzkapitalismus, Mobilitätskapitalismus, Hyperindustriekapitalismus und Informationskapitalismus darstellt.

Und diese Dimensionen des Kapitalismus sind miteinander verschränkt und greifen ineinander. Digitale Unternehmen sind ein gutes Beispiel dafür: Viele dieser Konzerne werden von Venture-Kapital-Unternehmen finanziert, wodurch sich eine Krisenanfälligkeit der Internetökonomie auf Finanzmärkten ergibt, da diese Finanzinvestitionen oft sehr risikoreich sind. Digitale Medien können ohne Energieversorgung nicht existieren. Das globale Internet konsumiert derzeit ca. $10 \%$ der global verbrauchten Energie (vgl. De Decker 2015). In Zeiten von Big Data und Serverfarmen steigt dieser Anteil an. Die Digitalisierung hat eine Flexibilisierung und globale Mobilisierung von Waren, Personen und Information unterstützt und vermittelt.

Die Krisenanfälligkeit des Kapitalismus ist ein fünfter wichtiger Aspekt der Kritik der Politischen Ökonomie. Der Kapitalismus ist ein inhärent krisenbehaftetes System. „Die widerspruchsvolle Bewegung der kapitalistischen Gesellschaft macht sich dem praktischen Bourgeois am schlagendsten fühlbar in den Wechselfällen des periodischen Zyklus, den die moderne Industrie durchläuft, und deren Gipfelpunkt - die allgemeine Krise.“ (Marx 1867, S. 28) Die neue Weltwirtschaftskrise und ihre Folgen verdeutlichen die Wichtigkeit der objektiven Dialektik des Kapitalismus, also seiner Krisenanfälligkeit. Eine komplexe Kombination aus Lohnstagnation, Klassenkampf von oben, Finanzialisierung, Prekarisierung und dem Anstieg der technischen und organischen Zusammensetzung des Kapitals durch Computerisierung, Informatisierung und Automatisierung hat Widersprüche im Laufe der Jahrzehnte heranreifen lassen, die sich dann in der Krise aufgehoben haben und nunmehr ausdrücken. Die kapitalistische Internetökonomie ist auf Grund ihrer Finanzialisierung ein stark krisenanfälliger Bereich des Kapitalismus. Die Dot-com-Krise im Jahr 2000 hat dies verdeutlicht. Auch der Bereich der Ökonomie sozialer Medien unterliegt ähnlichen Finanzialisierungstendenzen.

Die Dialektik von Technik und Gesellschaft ist ein sechster bedeutender Aspekt der Kritik der Politischen Ökonomie. Marx hat diese Dimension im sogenannten Maschinenfragment in den Grundrissen sowie im dreizehnten Kapitel des ersten Bandes des Kapitals (Maschinerie und große Industrie) dargestellt. Er schreibt dort zum Beispiel, dass ,die Maschinerie an sich betrachtet die Arbeitszeit verkürzt,

\footnotetext{
2 Datenquelle: Forbes 2000 Liste für das Jahr 2016, https://www.forbes.com/global2000/list/, zugegriffen
} am 25. März 2017. 
während sie kapitalistisch angewandt den Arbeitstag verlängert, an sich die Arbeit erleichtert, kapitalistisch angewandt ihre Intensität steigert, an sich ein Sieg des Menschen über die Naturkraft ist, kapitalistisch angewandt den Menschen durch die Naturkraft unterjocht, an sich den Reichtum des Produzenten vermehrt, kapitalistisch angewandt ihn verpaupert usw.“ (Marx 1867, S. 465)

Unter „Dialektik der Technik“ ist zu verstehen, dass sich die Technik in einer Klassengesellschaft unter Einfluss der bestehenden und sich entwickelnden gesellschaftlichen Widersprüche ebenfalls widersprüchlich entwickelt. Zugleich ist die Technik nicht nur gesellschaftlich produziert, sondern hat auch unvorhersehbare interne Widersprüche und Entwicklungsdynamiken. Dies betrifft vor allem hochkomplexe Systeme, die ein Katastrophenrisiko beinhalten. Kommunikationstechnologien und anderen Technologien entwickeln sich weder mit Notwendigkeit in bestimmter Weise, noch ist ihre Entwicklung völlig zufällig. Vielmehr spielt die Dialektik von Zufall und Notwendigkeit eine Rolle. Raymond Williams, der sein Leben lang ein von Marx beeinflusster Theoretiker war, hat in seiner Kritik an Marshall McLuhans Technikdeterminismus betont, dass die Technik keine ,automatische Kraft ist, die neue Lebensarten schafft" (Williams 2003/1974, S. 6). Vielmehr spielen menschliche Intentionen und Handlungen, die von bestimmten gesellschaftlichen Bedingungen beeinflusst werden, eine bedeutende Rolle.

Der siebente wichtige Aspekt der Kritik der Politischen Ökonomie besteht in der Auseinandersetzung mit der Wissensbasierung der Gesellschaft und des Kapitalismus. Marx hat im „Maschinenfragment“ den Begriff des General Intellect eingeführt. Damit bezeichnet er das ,,allgemeine gesellschaftliche Wissen, knowledge“ (Marx 1857/58, S. 608). Er beschreibt einen Zustand, in dem ,das allgemeine gesellschaftliche Wissen, knowledge, zur unmittelbaren Produktivkraft geworden ist und daher die Bedingungen des gesellschaftlichen Lebensprozesses selbst unter die Kontrolle des general intellect gekommen und ihm gemäß umgeschaffen sind“. Einerseits betont Marx mit dem Begriff des General Intellect, dass Wissen, Technik und Wissenschaft allgemeine Bedingungen der modernen Wirtschafft darstellen, die in viele Produktionsprozesse zugleich einfließen und von vielen Menschen kooperativ geschaffen und genutzt werden. Andererseits antizipiert er mit dieser Kategorie die Informatisierung der Ökonomie.

In Zusammenhang mit dem General Intellect steht auch der Umstand, dass sich Marx mit den neuen Medien seiner Zeit (insbesondere dem Telegrafen) und deren Rolle in der Gesellschaft beschäftige. Der achte Aspekt ist daher, dass die Beschäftigung mit Marx historische, theoretische und methodische Einsichten für die Technikund Mediensoziologie und die Analyse der Kommunikationsmittel und der menschlichen Kommunikation bietet. So beschrieb Marx zum Beispiel Kommunikationssysteme, ,worin jeder einzelne sich Auskunft über die Tätigkeit aller andren verschafft“ und „Verhältnisse und Verbindungen“ hergestellt werden (Marx 1857/1858, S. 94). Diese Formulierung zeigt verblüffende Ähnlichkeit mit den kommunikativen Eigenschaften des Internets, wurde aber in den 1850er-Jahren geschrieben, also mehr als 100 Jahre vor der technischen Schaffung des Internets.

Der neunte Aspekt der Kritik der Politischen Ökonomie, der heute von Bedeutung ist, ist der Widerspruch zwischen Produktivkräften und Produktionsverhältnissen (vgl. Marx 1894, S. 274-275). Durch die kapitalistische Entwicklung entstehen 
neue Formen der Kooperation und Kooperationstechnologien, die Grundlagen für neue Gemeingüter darstellen, zugleich aber als kapitalistisches Privateigentum Mittel der Herrschaftsausübung sind. Dieser Widerspruch zeigt sich heute deutlich bei vernetzten digitalen Technologien, die zugleich neue Weisen der Kommodifizierung und Ausbeutung hervorbringen und sich der Kommodifizierung widersetzen und Sphären der nichtkapitalistischen Kommunikation begründen können. Der Widerspruch von Produktivkräften und Klassenverhältnissen äußert sich im digitalen Kapitalismus als Widerspruch zwischen digitalen Gemeingütern und digitalen Waren. Negri (2017, S. 25) spricht in diesem Zusammenhang davon, dass die Digitalisierung geprägt ist durch einen „Antagonismus der gesellschaftlichen Kooperation des Proletariats [der gesellschaftliche Arbeiter] und dem (wirtschaftlichen und politischen) Kommando des Kapitals“.

Beim zehnten wichtigen Aspekt der Kritik der Politischen Ökonomie handelt es sich um Beiträge zur Kommunikationstheorie. „Erst durch die Beziehung auf den Menschen Paul als seinesgleichen bezieht sich der Mensch Peter auf sich selbst als Mensch." (Marx 1867, S. 67) Durch den Kommunikationsprozess beziehen die Menschen in komplexer Weise ihre gedanklichen Reflexionen wechselseitig aufeinander, was zu kognitiven Veränderungen führt und die Sozialität des Menschen und die Gesellschaftlichkeit produziert und reproduziert. Wichtige Beiträge zur Kommunikationstheorie wurden in der auf Marx beruhenden Tradition u. a. durch Georg Lukács' Ontologie des gesellschaftlichen Seins und die Arbeiten von Raymond Williams, Ferruccio Rossi-Landi, Theodor W. Adorno, Herbert Marcuse, Lev Vygotsky und Valentin Vološinov geleistet (vgl. Fuchs 2016a). Werden diese theoretischen Ansätze zusammengedacht, so ergibt sich dadurch eine kommunikationsmaterialistische Alternative zur dualistischen kritischen Kommunikationstheorie von Jürgen Habermas und zur instrumentellen Kommunikationssystemtheorie von Niklas Luhmann.

Der elfte aktuelle Aspekt hat damit zu tun, dass Marx im Basis/Überbau-Problem die Frage nach dem Zusammenhang von Wirtschaft und Gesellschaft, Werktätigkeit und Kommunikation, Arbeit und Ideologie, Körper und Geist, körperlicher und geistiger Arbeit, Produktion und Reproduktion, Natur und Gesellschaft stellt. Raymond Williams betont in seinem Ansatz des kulturellen Materialismus Marx' Einsicht, dass es sich bei der Gesellschaft um einen Zusammenhang der sozialen Produktion der Sozialität des Menschen handelt (vgl. Williams 1977). Die durch Kommunikation vermittelte soziale Produktion ist ein identisches Moment aller sozialen Systeme und gesellschaftlichen Teilbereiche. Es handelt um das ökonomische Moment des Sozialen. Zugleich haben aber alle sozialen Systeme und Bereiche emergente Qualitäten, die sie von anderen Systemen und Bereichen und dem rein wirtschaftlichen Aspekt unterscheiden und aufheben.

Der zwölfte Grund, warum die Kritik der Politischen Ökonomie heute Relevanz hat, ist die Rolle, die Ideologien und der Fetischcharakter der Waren in der heutigen Gesellschaft spielen. Unter dem Warenfetischismus versteht Marx, dass das „bestimmte gesellschaftliche Verhältnis der Menschen selbst [...] die phantasmagorische Form eines Verhältnisses von Dingen“ annimmt (Marx 1867, S. 86). Ideologie naturalisiert und normalisiert die Naturalisierung. Charakteristisch für die Ideologie im Kapitalismus ist heute die Ausbreitung neuer Nationalismen kombiniert mit 
neuem Rassismus und Xenophobie, die sich vor allem gegen Arbeitsmigranten und Flüchtlinge richten. Nationalismus ist eine Ideologie, die eine fiktive Ethnizität (vgl. Balibar und Wallerstein 1991) konstruiert und eine fiktive Einheit des Interesses von Kapital und Arbeit in einem Nationalinteresse proklamiert. Von den komplexen Ursachen und Zusammenhängen gesellschaftlicher Probleme wird dadurch abgelenkt. Der Nationalismus ist eine politische Form des Fetischismus, in der die Nation und ein nationales und völkisches Kollektiv fetischiert werden. Der Nationalismus gibt vor, ,alle Klassen zu vereinigen durch die Wiederbelebung des Trugbilds des nationalen Ruhms“ (Marx 1871, S. 338).

Der dreizehnte Aspekt der Kritik der Politischen Ökonomie, der heute wichtig ist, ist die Rolle der gesellschaftlichen Kämpfe und Klassenkämpfe bei gesellschaftlichem Wandel. Marx sieht dabei eine historische Dialektik von Zufall und Notwendigkeit, Praxis und Strukturbedingungen. Wenn er sagt, dass die Menschen ihre „eigene Geschichte“ machen, aber ,nicht aus freien Stücken, nicht unter selbstgewählten, sondern unter unmittelbar vorgefundenen, gegebenen und überlieferten Umständen“ (Marx 1852, S. 115), so hat diese Einsicht heute praktische Relevanz. Heute gibt es im Kontext der Diskussion des gesellschaftlichen Wandels Diskussionen darüber, welche Rolle soziale Medien in Rebellionen, Protesten, Revolutionen und sozialen Bewegungen spielen. Es stellt sich dabei die Frage, ob die Menschen, die Krisen oder die Medientechnologien Geschichte machen. Um diese Frage zu beantworten, braucht man ein theoretisches Modell des gesellschaftlichen Wandels, das die Dialektik von Strukturen und Praxis und den Einfluss der Kommunikationstechnologien berücksichtigt. Die Orientierung der Kritik der Politischen Ökonomie an gesellschaftlichen Kämpfen beruht auf einem praktischen Humanismus, der Verhältnisse in Frage stellt, in denen der Mensch ,ein erniedrigtes, ein geknechtetes, ein verlassenes, ein verächtliches Wesen ist“ (Marx 1844, S. 385).

Der vierzehnte Aspekt, den die auf Marx beruhende Kapitalismuskritik betont, ist die Notwendigkeit und Wichtigkeit von demokratischen Alternativen zu Kapitalismus und Kapitalakkumulation. Bei Marx geht es um die Ausweitung der Demokratie von der Politik auf die Wirtschaft. Im Bereich der Medien verweist dies auf die nicht-kommerziellen, nicht-profitorientierten, am kommunikativen Gemeingut orientierten Alternativmedien. Im Bereich des akademischen Publizierens haben zum Beispiel Non-Profit-Open-Access-Zeitschriften an größerer Bedeutung gewonnen, die die Kapitalakkumulationsstrategien kommerzieller Verlag in Frage stellen (vgl. Fuchs und Sandoval 2013; Knoche 2014).

Die Diskussion der Relevanz der Kritik der Politischen Ökonomie könnte noch lange fortgesetzt werden, da die Anzahl ihrer Vertreterinnen und Vertreter mit wichtigen Ideen in dieser Theorietradition sehr groß ist.

\section{Ein Anwendungsbeispiel: soziale Bewegungen und Alternativmedien im Kapitalismus}

Die Kritik der Politischen Ökonomie hat eine geisteswissenschaftliche Tradition in Form der dialektischen Sozialphilosophie und eine gesellschaftswissenschaftliche Tradition in Form der kritischen Gesellschaftsanalyse. Praktisch gesehen kann man 
diese beiden Dimensionen nicht strikt trennen, sie treten oft kombiniert als theoriegeleitete empirische Kapitalismus- und Gesellschaftskritik auf.

Die Erforschung der Kommunikation sozialer Bewegungen hat sich international als Teilaspekt der Medien- und Kommunikationswissenschaft etabliert. Während des Arabischen Frühlings und der Occupy-Bewegungen wurde viel darüber diskutiert, welche Rolle soziale Medien in Protesten und Revolutionen spielen (vgl. Fuchs 2014b, 2017b). Der Großteil der zu solchen Fragen publizierten Arbeiten beschäftigte sich rein mikrosoziologisch mit der Frage, wie soziale Bewegungen kommunizieren, ohne den breiteren makrosoziologischen Kontext, in dem diese Bewegungen operieren, nämlich das kapitalistische Weltsystem, zu berücksichtigen. Die Studie OccupyMedia! The Occupy Movement and Social Media in Crisis Capitalism (vgl. Fuchs 2014b) war hingegen als eine Arbeit angelegt, die die Kritik der Politischen Ökonomie als empirischen Ansatz verwendete. Methodisch wurde eine Onlineumfrage durchgeführt, an der 418 Occupy-Aktivistinnen und -Aktivisten teilnahmen.

Eines der Ergebnisse war, dass weder Onlinekommunikation noch Face-to-FaceKommunikation allein die entscheidende Kommunikationsform von Aktivisten, die öffentliche Plätze besetzen, darstellen, sondern dass eine Dialektik besteht: Je aktiver Aktivisten in der Bewegung sind, desto größer ist ihr soziales Protestnetzwerk und desto mehr verwenden sie tendenziell Online- und Offlinekommunikation, die sich wechselseitig verstärken, für interne Bewegungskommunikation und externe, öffentliche Mobilisierungskommunikation. Bewegungsinterne und -externe Kommunikation stehen nun aber im Kontext kapitalistischer und staatlicher Macht, gegen die sich solche Bewegungen meist wenden. Es stellt sich daher die Frage, wie kapitalistische und staatliche Kommunikationskontrolle soziale Bewegungen beeinflusst. Die Umfrage verdeutlichte einen Widerspruch zwischen öffentlicher Kommunikation und Kommunikationskontrolle auf kapitalistischen sozialen Medien: Aktivistinnen und Aktivisten sehen es als großen Vorteil, dass diese Onlineplattformen hohe Nutzerzahlen haben, da dadurch die Erreichung einer breiten Öffentlichkeit ermöglicht wird. Da die Unternehmen (wie Facebook und Twitter), die diese Plattformen betreiben, zu „den $1 \%$ " gehören und einige davon, wie Edward Snowdens Enthüllungen gezeigt haben, in den überwachungsindustriellen Internetkomplex involviert sind, besteht für Protestbewegungen das Problem, dass die Nutzung dieser Plattformen mit bewusster oder algorithmischer Zensur sowie staatlicher Überwachung ihrer Kommunikation verbunden sein kann.

Ein Widerspruch zeigte sich auch hinsichtlich der Nutzung alternativer, nichtkommerzieller sozialer Medien: Einerseits bieten diese mehr Autonomie und Schutz vor Staat und Kapital, andererseits sind sie relativ unbekannt, erreichen daher nur wenig Menschen und sind zusätzlich mit der für den Kapitalismus typischen Ressourcenungleichheit konfrontiert, die dazu führt, dass Alternativmedien oft wenig Sichtbarkeit, Geld, Personal, Reputation oder Einfluss haben, auf freiwilliger Selbstausbeutung und prekärer Arbeit beruhen, auf Grund von Ressourcenmangel verschwinden oder kapitalistisch werden.

Danach befragt, wie mit dem Widerspruch der Alternativmedien im Kapitalismus umgegangen werden sollte, befürworteten 54,7\% das Modell freiwilliger Spenden, 9,4\% Nutzungsgebühren auf Non-Profit-Basis, 8,0\% personalisierte Werbung und 
7,0\% staatliche Subventionen (vgl. Fuchs 2014b). Spenden- und CrowdfundingModelle sind aber wiederum mit dem Widerspruch konfrontiert, dass die Spenden für alternative Projekte zum großen Teil von Aktivistinnen und Aktivisten kommen, die meist keine Millionäre sind, sondern oft prekär Beschäftigte, wodurch solche Modelle leicht finanziell instabil werden können. Der grundsätzliche kapitalistische Widerspruch besteht in diesem Zusammenhang zwischen Medienkonzernen wie Google und Facebook, die Milliarden schöpfen und dafür kaum Steuern bezahlen, und der Dauerkrise der kritischen Alternativmedien. Dieser Widerspruch kann im Kapitalismus nicht gelöst werden. Es bedarf radikaler Reformen, die Kapital, Profit und Werbung besteuern und die so erzielten Erlöse (z. B. in der Form eines Grundeinkommens, von partizipativem Budgeting für nichtkommerzielle Alternativmedien und nichtkommerzielle Projekte etc.) für öffentliche und kritische Zwecke nützen. Das Grundproblem ist die strukturelle Beschränkung und Limitierung der kommunikativen Demokratie im Kapitalismus.

Die derzeit größte Herausforderung für die Erforschung der politischen Kommunikation ist der massiv ansteigende Nationalismus und Rechtspopulismus, was am Beispiel Donald Trumps in den USA deutlich wird. In diesem Zusammenhang spielen soziale Medien, Reality TV und andere populäre Medien eine wichtige Rolle. Traditionen der Kritik der Politischen Ökonomie, wie etwa die Theorie der autoritären Persönlichkeit der Frankfurter Schule (Franz L. Neumann, Erich Fromm, Theodor W. Adorno, Leo Löwenthal, Herbert Marcuse etc.), stellen in diesem Kontext einen wichtigen Ansatzpunkt dar.

\section{Schlussfolgerungen}

Die Kritik der Politischen Ökonomie ist ein fruchtbarer Ansatz zur empirischen und theoretischen Analyse und zur Aufklärung zeitgenössischer Kommunikation, der eine wahrhafte Praxisrelevanz hat.

Ich habe in diesem Beitrag zu zeigen versucht, dass in der Medien- und Kommunikationswissenschaft die ,Angst der WissenschaftlerInnen vor Marx [...] unberechtigt" (Knoche 2005, S. 411) ist. Ganz im Gegenteil: Wir sind heute in einer gesellschaftlichen Situation, in der die Krise des Kapitalismus mit einer allgemeinen Legitimationskrise verbunden ist und autoritäre Bewegungen und autoritärer Kapitalismus die Grundfesten der Demokratie angreifen. Neoliberaler Kapitalismus schlägt heute in einer negativen Dialektik tendenziell in autoritären Kapitalismus um. Kommunikationsplattformen, -strategien und Ideologien spielen in dieser turbulenten gesellschaftlichen Situation eine wichtige Rolle. Die intellektuelle Erbschaft von Karl Marx, Max Horkheimer, Theodor W. Adorno, Georg Lukács, Raymond Williams und anderer in dieser kritischen Tradition stehenden Wissenschaftler und Wissenschaftlerinnen ermöglicht eine praxisrelevante, gesellschaftskritische Kommunikationswissenschaft, die heutige Krisen und Formen von Autoritarismus besser verstehen kann, sowie Ansatzpunkte für eine kritische, öffentliche Gesellschaftswissenschaft (vgl. Aulenbacher et al. 2017), die in öffentliche Diskurse interveniert.

Der Fall Schamberger verdeutlicht, dass Vertreter der Kritik der Politischen Ökonomie der Medien/Kommunikation im deutschen Sprachraum immer noch ,berech- 
tigte Angst“" haben müssen, ,als Marxist zu gelten“ (Knoche 2005, S. 411). Theorien des Kapitalismus, der autoritären Persönlichkeitsstruktur, der Krisen, der Ideologie, der kritischen Kommunikation usw., wie sie sich in der Kritik der Politischen Ökonomie finden, sind heute zentral für die Gesellschafts- und Kommunikationswissenschaft. Die im Frühjahr 2017 erfolgte Gründung des Netzwerks Kritische Kommunikationswissenschaft (siehe https://dimbb.de/wp-content/uploads/2017/03/ Netzwerk-KriKoWi_Aufruf-zur-Gr\%C3\%BCndung.pdf) und die Einrichtung einer dazugehörigen Mailingliste (https://lists.riseup.net/www/info/krikowi) ist in diesem Kontext eine erfreuliche Entwicklung.

Open Access Dieser Artikel wird unter der Creative Commons Namensnennung 4.0 International Lizenz (http://creativecommons.org/licenses/by/4.0/deed.de) veröffentlicht, welche die Nutzung, Vervielfältigung, Bearbeitung, Verbreitung und Wiedergabe in jeglichem Medium und Format erlaubt, sofern Sie den/die ursprünglichen Autor(en) und die Quelle ordnungsgemäß nennen, einen Link zur Creative Commons Lizenz beifügen und angeben, ob Änderungen vorgenommen wurden.

\section{Literatur}

Aulenbacher, B., Burawoy, M., Dörre, K., \& Sittel, J. (Hrsg.). (2017). Öffentliche Soziologie: Wissenschaft im Dialog mit der Gesellschaft. Frankfurt am Main: Campus.

Balibar, É., \& Wallerstein, I. (1991). Race, nation, class. London: Verso.

Bidet, J., \& Kouvelakis, S. (Hrsg.). (2008). Critical companion to contemporary marxism. Leiden: Brill.

Bönkost, J. (2011). Im Schatten des Aufbruchs. Das erste Berufsverbot für Horst Holzer und die Uni Bremen. Grundrisse, 39, 29-37.

Dalla Costa, M., \& James, S. (1973). The power of women and the subversion of community (2. Aufl.). Bristol: Falling Wall.

De Decker, K. (2015). Why we need a speed limit for the Internet. http://www.lowtechmagazine.com/ 2015/10/can-the-internet-run-on-renewable-energy.html (Erstellt: 19. Okt 2015). Zugegriffen: 08. Juni 2017

Federici, S. (2012). Revolution at point zero. Housework, reproduction and feminist struggle. Oakland: PM Press.

Fuchs, C. (2008). Internet and society: social theory in the information age. New York: Routledge.

Fuchs, C. (2011). Foundations of critical media and information studies. London: Routledge.

Fuchs, C. (2014a). Digital labour and Karl Marx. New York: Routledge.

Fuchs, C. (2014b). OccupyMedia! The Occupy movement and social media in crisis capitalism. Winchester: Zero Books.

Fuchs, C. (2015). Culture and economy in the age of social media. New York: Routledge.

Fuchs, C. (2016a). Critical theory of communication. New readings of Lukács, Adorno, Marcuse, Honneth and Habermas in the age of the Internet. London: University of Westminster Press.

Fuchs, C. (2016b). Reading Marx in the information age: a media and communication studies perspective on Capital Volume I. New York: Routledge.

Fuchs, C. (2017a). Marx lesen im Informationszeitalter: Eine medien- und kommunikationswissenschaftliche Perspektive auf „Das Kapital. Band 1“. Münster: Unrast.

Fuchs, C. (2017b). Social media: a critical introduction (2. Aufl.). London: SAGE.

Fuchs, C., \& Mosco, V. (2012). Marx is back - the Importance of Marxist theory and research for critical communication studies today. triple C: Communication, Capitalism \& Critique, 10(2), 127-632.

Fuchs, C., \& Mosco, V. (Hrsg.). (2017a). Marx and the political economy of the media. Chicago: Haymarket Books.

Fuchs, C., \& Mosco, V. (Hrsg.). (2017b). Marx in the age of digital capitalism. Chicago: Haymarket Books.

Fuchs, C., \& Sandoval, M. (2013). The diamond model of open access publishing: Why policy makers, scholars, universities, libraries, labour unions and the publishing world need to take non-commercial, non-profit open access serious. triple C: Communication, Capitalism \& Critique, 11(2), 428-443.

Hardy, J. (2014). Critical political economy of the media. An introduction. Abingdon: Routledge.

Harvey, D. (2005). Der neue Imperialismus. Hamburg: VSA. 
Holzer, H. (1971). Gescheiterte Aufklärung? Politik, Ökonomie und Kommunikation in der Bundesrepublik Deutschland. München: Piper.

Holzer, H. (1973). Kommunikationssoziologie. Reinbek bei Hamburg: Rowohlt.

Holzer, H. (1987). Kommunikation oder gesellschaftliche Arbeit? Zur Theorie des kommunikativen Handelns von Jürgen Habermas. Berlin: Akademie.

Holzer, H. (1994). Medienkommunikation. Opladen: Westdeutscher Verlag.

Jhally, S. (2016). Stuart Hall: the last interview. Cultural Studies, 30(2), 332-345.

Knoche, M. (2002). Kommunikationswissenschaftliche Medienökonomie als Kritik der Politischen Ökonomie der Medien. In G. Siegert (Hrsg.), Medienökonomie in der Kommunikationswissenschaft. Bedeutung, Grundfragen und Entwicklungsperspektiven. Manfred Knoche zum 60. Geburtstag (S. 101-109). Münster: LIT.

Knoche, M. (2005). Medienökonomische Theorie und Ideologie im Kapitalismus. In C.-M. Ridder et al. (Hrsg.), Bausteine einer Theorie des öffentlich-rechtlichen Rundfunks (S. 406-435). Wiesbaden: VS.

Knoche, M. (2014). Emanzipatorische Transformation der Wissenschaftskommunikation statt Irrweg Verlags-TOLL OPEN ACCESS. Medien Journal, 38(4), 76-78. doi:10.24989/mj.v38i4.91.

Marx, K. (1842). Debatten über Preßfreiheit und Publikation der Landständischen Verhandlungen. Von einem Rheinländer. Marx-Engels-Werke, Bd. 1 (S. 28-77). Berlin: Dietz.

Marx, K. (1844). Zur Kritik der Hegelschen Rechtsphilosophie. Marx-Engels-Werke, Bd. 1 (S. 378-391). Berlin: Dietz.

Marx, K. (1852). Der achtzehnte Brumaire des Louis Bonaparte. Marx-Engels-Werke, Bd. 8 (S. 111-207). Berlin: Dietz.

Marx, K. (1857/1858). Grundrisse der Kritik der politischen Ökonomie. Berlin: Dietz.

Marx, K. (1867). Das Kapital. Kritik der politischen Ökonomie. Bd 1. Marx-Engels-Werke, Bd. 23. Berlin: Dietz.

Marx, K. (1871). Der Bürgerkrieg in Frankreich. Marx-Engels-Werke, Bd. 1 (S. 313-365). Berlin: Dietz.

Marx, K. (1894). Das Kapital. Kritik der politischen Ökonomie. Bd 1. Marx-Engels-Werke, Bd. 25. Berlin: Dietz.

Marx, K., \& Engels, F. (1848). Manifest der Kommunistischen Partei. Marx-Engels-Werke, Bd. 4 (S. 459-493). Berlin: Dietz.

Mosco, V. (2009). The political economy of communication (2. Aufl.). London: SAGE.

Murdock, G., \& Golding, P. (1974). For a political economy of mass communications. In P. Golding \& G. Murdock (Hrsg.), The political economy of the media (Bd. I, S. 3-32). Cheltenham: Edward Elgar.

Murdock, G., \& Golding, P. (2005). Culture, communications and polticial economy. In J. Curran \& M. Gurevitch (Hrsg.), Mass media and society (4. Aufl. S. 60-83). London: Hodder Arnold.

Negri, A. (1982/1988). Archaeology and project: The mass worker and the social worker. In Revolution retrieved: Selected writings on Marx, Keynes, capitalist crisis \& new social subjects 1967-83 (S. 199-228). London: Red Notes.

Negri, A. (2017). Marx and Foucault. Cambridge: Polity.

Schamberger, K. (2017). tripleC-Interview mit Kerem Schamberger über Berufsverbote, linke Kommunikationswissenschaft und Kritik an der deutschen Wissenschaftslandschaft. tripleC: Communication, Captialism \& Critique, 15(1), 91-99.

Scheu, A. M. (2012). Adornos Erben in der Kommunikationswissenschaft. Eine Verdrängungsgeschichte? Köln: Halem.

Smythe, D. W. (1977). Communications: blindspot of Western Marxism. Canadian Journal of Political and Social Theory, 1(3), 1-27.

Wasko, J. (2014). The study of political economy of the media in the twenty-first century. International Journal of Media \& Cultural Politics, 10(3), 259-271.

Williams, R. (1977). Marxism and literature. Oxford: Oxford University Press.

Williams, R. (2003/1974). Television. London: Routledge.

Winseck, D. (2011). The political economies of media and the transformation of the global media industries. In D. Winseck \& D. Y. Jin (Hrsg.), The political economies of media. The transformation of the global media industries (S. 3-48). London: Bloomsbury Academic.

Wright, E. O. (1997). Class counts: comparative studies in class analysis. Cambridge: Cambridge University Press.

Prof. Dr. Christian Fuchs ist Professor für Medien- und Kommunikationswissenschaft an der University of Westminster in London. 